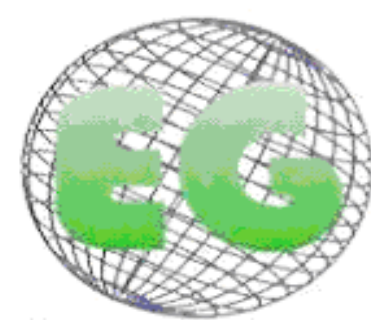

ISSN 1695-6141 $N^{\circ} 22$
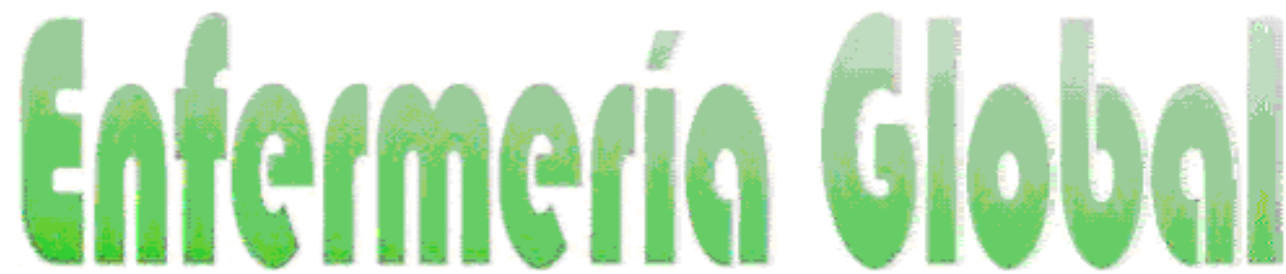

Revista electrónica trimestral de Enfermería

Abril 2011

www.um.es/egloball

\title{
PERCEPCIÓN DE LA ASISTENCIA SANITARIA AL EMBARAZO Y PARTO EN MUJERES CON DISCAPACIDAD DE LA MOVILIDAD EN LA REGIÓN DE MURCIA
}

PERCEPTION OF MEDICAL CARE DURING PREGNANCY AND BIRTH IN WOMEN WITH MOBILITY IMPAIRMENT IN THE REGION OF MURCIA

\author{
*Ballesteros Meseguer, C., "*Marín, NT., ${ }^{* * *}$ Martínez-Espejo, MD., ${ }^{* * * * P i n a}$ \\ Roche, F. \\ *Matrona. Profesora Asociada Clínica Departamento de Enfermería. ${ }^{* *}$ Profesora Titular Departamento de \\ Fisiología. ***Profesora Titular Departamento de Enfermería. Facultad de Enfermería. Universidad de Murcia. \\ Palabras clave: Maternidad; Discapacidad; Enfermería. \\ Keywords: Motherhood; Disability; Nursing
}

\section{RESUMEN}

Introducción. Según investigaciones previas, las mujeres con discapacidad se encuentran en una situación de especial desigualdad ante los servicios sanitarios por dificultades de accesibilidad, falta de información y las necesidades específicas que se derivan de su particular situación. Este trabajo tiene como objetivo analizar cómo perciben la asistencia sanitaria al embarazo y parto las mujeres con discapacidad de la Región de Murcia y constatar si se encuentran en una situación de especial desigualdad ante los servicios sanitarios durante su proceso de embarazo y parto.

Material y métodos. Se han realizado dos estudios. En el primero, de carácter cualitativo, se han entrevistado a cinco personas con discapacidad en proceso de embarazo y se ha repartido una encuesta de elaboración propia a 17 Asociaciones de Discapacitados de la Región de Murcia. En el segundo estudio, de carácter epidemiológico, se utilizó la base datos del servicio de Codificación de Historias Clínicas del Hospital Universitario Virgen de la Arrixaca de Murcia (HUVA). Se recogieron 3.335 partos, donde la mujer había sido diagnosticada con discapacidad entre el año 2005 hasta el 2009 .

Resultados y conclusiones. Los principales resultados de nuestro estudio han sido: a) no se ha constatado un aumento en la medicalización del parto entre mujeres con discapacidad y sin ella; b) las mujeres con discapacidad perciben una falta de sensibilidad en el trato de los profesionales de Sanidad, falta de respeto a su intimidad y sufren carencias de información y educación sanitaria; c) las Asociaciones para discapacitados no han recogido esta problemática entre los objetivos de su asociación. De este estudio podemos concluir que los embarazos en las mujeres con discapacidad considerados como "de alto riesgo", que son controlados en Atención Especializada exclusivamente por el obstetra, carecen de los beneficios que supone la Atención Primaria en cuanto a información, 
educación sanitaria y maternal proporcionada por la matrona e integrada dentro de un equipo multidisciplinar. Así mismo, sería recomendable que todos los profesionales sanitarios, que prestan sus servicios a este colectivo, fuesen debidamente formados en materia de discapacidad.

\section{ABSTRACT}

Introduction. According to previous research, women with disability are in a particular situation of inequality in health services due to difficulties of accessibility, lack of attention and specific needs which are derived from their particular situation. The goal of this essay is to analyze how these women with disability perceive the medical care received during their pregnancy and birth giving in the Region of Murcia and to verify if they are in a situation of inequality in health services during these processes.

Material and methods. Two different studies were conducted. In the first, following a qualitative approach, five people in a situation of inequality during their pregnancy were interviewed. A self-made questionnaire was also delivered to seventeen Associations of Disabled People in the Region of Murcia. In the second, following an epidemiological approach, data from the Clinical Stories Codification services from a Hospital in Murcia was used. Data from 3.335 births in which women had been diagnosed with a disability between 2005 and 2009 were collected.

Results and conclusions. The results of our study showed that: a) there was no increase in medical care during birth between women with disability and women without disability; b) women with disability perceive a lack of sensitivity in the treatment by health professionals; a lack of respect for her intimacy and a lack of information and health education. The Association of Disabled people has not included these problems among their main goals of the Association. From this study, we can conclude that pregnancies in women with disability considered as "high risk pregnancies" and which are controlled by Specialized Services exclusively by the obstetrician, lack the benefits of the Primary Attention regarding information and health and maternal education proportioned by the midwife, who is integrated in a multidisciplinary team. Likewise, it would be advisable that all health professionals, who render their services to this group were properly trained in disability matters.

\section{INTRODUCCIÓN}

La discapacidad es una realidad humana que ha sido percibida de manera distinta en diferentes periodos históricos y civilizaciones. De forma resumida, hemos ido revisando los diferentes modelos de concebir la discapacidad ${ }^{1}$ :

- Modelo Demonológico (Edad Media S VI-XV): La discapacidad se concebía como una posesión demoníaca, se la asociaba al mal, los discapacitados eran quemados, asesinados y encerrados.

- Modelo Organicista (1400-1500): Se cree que la discapacidad tiene causas orgánicas y se busca otorgar remedio a estos problemas.

- Modelo socioambiental (A partir de la II Guerra Mundial): La persona con discapacidad es un ser social que tiene que ser reincorporado a su medio.

- A partir de 1945-1955, La Organización de Naciones Unidas ${ }^{2}$, durante su primera década de trabajo en el ámbito de la discapacidad, se esforzó en proporcionar a los discapacitados una perspectiva de bienestar. Defendiendo los derechos humanos, aparece el Modelo Rehabilitador, que buscaba la rehabilitación social de los discapacitados físicos.

- De 1955-1970, surge una perspectiva de bienestar social. Se desinstitucionaliza a los discapacitados y se busca una participación de las personas con discapacidad en una sociedad integrada. Es el llamado Modelo de Integración.

- Ya desde 1970, y siempre desde la perspectiva de los derechos humanos, se busca la igualdad en derechos políticos y civiles que les permitan ser autosuficientes (en 
materia de educación, sanidad y empleo), es el Modelo de la Autonomía personal. Este modelo ha ido evolucionando hasta nuestros días buscando la plena integración en la sociedad de las personas con discapacidad y con miras hacia un futuro en el que se construya una "sociedad para todos".

- A lo largo del siglo XX, la discapacidad siempre se ligaba únicamente a una condición o función que se consideraba deteriorada respecto a la situación general de un individuo. Pero, en el año 1980, la Organización Mundial de la Salud, presentó un documento, «Clasificación Internacional de Deficiencias, Discapacidades y Minusvalías» $(\mathrm{CIDMM})^{3}$, que sirvió para definir a las personas con discapacidad, no por las causas de sus minusvalías, sino por las consecuencias que éstas les han generado. Así, la discapacidad pasa a ser considerada como un término general que abarca las deficiencias, las limitaciones de la actividad y las restricciones de la participación. Las deficiencias son problemas que afectan a una estructura o función corporal; las limitaciones de la actividad son dificultades para ejecutar acciones o tareas, y las restricciones de la participación son problemas para participar en situaciones vitales. Por consiguiente, la discapacidad es un fenómeno complejo que refleja una interacción entre las características del organismo humano y las características de la sociedad en la que vive. $(\mathrm{OMS})^{3}$. A partir de aquí, la discapacidad comenzó a ser considerada a partir de los derechos humanos. Iniciándose una búsqueda para mantener la discapacidad dentro de la sociedad, con actitudes inclusivas y normas de accesibilidad.

Habría que esperar al año 2006, en el que la Organización de Naciones Unidas acordó la Convención sobre los Derechos de Personas con Discapacidad ${ }^{4}$, para proteger y reforzar los derechos y la igualdad de oportunidades de los 650 millones de personas con discapacidad en todo el mundo. En España, esta convención originó un instrumento de Ratificación ${ }^{5}$ de estos derechos humanos, donde se define la discapacidad como un concepto que evoluciona y que resulta de la interacción entre las personas con deficiencias y las barreras debidas a la actitud y al entorno que evitan su participación plena y efectiva en la sociedad, en igualdad de condiciones con las demás. Se trata, entonces, de apostar por la mejora humana y profesional, que nos permita asumir una adecuada atención a la diversidad, poniendo la ciencia en función de garantizar la calidad de vida de todos los miembros de nuestra sociedad, sin distinción de raza, religión, sexo, edad, y por supuesto, sin distinción de la condición de salud.

Aunque como hemos comentado se ha avanzando mucho en materia de discapacidad, las diferencias de género siguen siendo palpables en este sector, a pesar de que las mujeres son mayoría entre la población de discapacitados ${ }^{6}$. Así, temas tan importantes para la mujer como su salud sexual y reproductiva, aún son asignaturas pendientes para muchas de ellas. La maternidad, en las mujeres con discapacidad, es un derecho reconocido. Sin embargo, ser madre con una discapacidad supone para la mujer una tarea difícil, en la que a las dificultades propias de la maternidad, hay que sumarle las inherentes a su discapacidad y las derivadas de la no aceptación social. Según Peláez ${ }^{7}$, la sociedad espera de ellas que no tengan hijos.

La doctora Welner ${ }^{8}$, especialista en Obstetricia y Ginecología de Georgetown Escuela Universitaria de Medicina de Washington DC., fue pionera en el ámbito de la asistencia ginecológica a las mujeres con discapacidad. Lo que la hacía especial era que comenzó a ver la medicina desde el lado del paciente. Observó cómo, tras su discapacidad, se cuestionaba constantemente su condición de persona competente y eso le hizo tomar la determinación de cambiar la atención médica para las mujeres con discapacidad. Para 
Welner, las mujeres con discapacidad son mujeres, en primer lugar, y tienen las mismas necesidades de atención de salud reproductiva que las mujeres sin discapacidad.

Cada vez son más las mujeres con discapacidad que están interesadas en convertirse en madres y criar a los hijos. Sin embargo, hay estudios que refieren que los profesionales de la salud no están familiarizados con las necesidades de este grupo de mujeres y pasan por alto las cuestiones importantes que hacen la diferencia entre las experiencias positivas y negativas $^{9}$. Según el Informe Ejecutivo, de 2009, del Comité Español de Representantes de Personas con Discapacidad ${ }^{10}$ (en adelante CERMI), las mujeres con discapacidad se encuentran en una situación de especial desigualdad ante los servicios sanitarios por dificultades de accesibilidad a los servicios ginecológicos, escasa e inadecuada respuesta a las necesidades de información y las necesidades específicas que se derivan de su particular situación. Otros estudios, concluyen que existen reticencias para atender estos embarazos, bajo el pretexto de considerarlos de alto riesgo; por lo que se realiza una práctica mayoritaria de cesáreas, etc. ${ }^{11-12}$

La importancia de este problema radica en que las mujeres con discapacidad, dentro del grupo de las personas con discapacidad, es el que sufre una mayor discriminación, pues al factor de relegación por una minusvalía, se añade el de género, común en todas las esferas de nuestra sociedad. Incluso me atrevería a mencionar una tercera discriminación, o causa de relegación social, al entender la sociedad actual que la mujer con discapacidad no cumple con los estereotipos sociales actuales de "buena" madre. Así, la sociedad espera de ellas que no tengan hijos, nos recoge en su libro Peláez ${ }^{7}$, sin embargo muchas de ellas anhelan tenerlos tanto como muchas otras jóvenes. ¿Qué ocurre cuando una mujer no satisface alguna de las funciones maternales que la sociedad espera de ella?, sin duda alguna, que se siente culpable, pero además será juzgada por gran parte de su entorno como una mala madre. ${ }^{13}$

La accesibilidad debe ser promovida por los profesionales sanitarios, a través de ciertas facilidades que ayudan a salvar los obstáculos del entorno, logrando que las discapacitadas puedan realizar la misma acción que pudiera llevar a cabo una persona sin ningún tipo de discapacidad. Los profesionales sanitarios que prestamos asistencia a las mujeres con discapacidad, formamos parte de esta sociedad que se cuestiona la capacidad de estas mujeres para ser madres, por tanto, nuestra actuación puede verse influenciada por los estereotipos sociales.

Por otro lado, para poder informar a la mujer con discapacidad, primero deberíamos estar informados los profesionales sanitarios acerca de la discapacidad que presenta la mujer y cómo va a repercutir en el normal desarrollo de la gestación.

Estas tres premisas, accesibilidad, influencia de los estereotipos sociales en la actuación profesional sanitaria y falta de información a la mujer con discapacidad, son las que tratamos de poner de manifiesto con la investigación, de forma que podamos obtener una visión más profunda de los cuidados que prestamos durante la asistencia sanitaria al embarazo y parto en la mujer con discapacidad en la Región de Murcia, con la finalidad de poder mejorar aquellos aspectos que impidan que la mujer con discapacidad viva su maternidad de forma positiva y con total normalidad.

El Objetivo General de la realización de este estudio es analizar cómo perciben la asistencia sanitaria al embarazo y parto las mujeres con discapacidad de la Región de Murcia y constatar si, como afirma el Informe Ejecutivo CERMI ${ }^{10}$, se encuentran en una situación de especial desigualdad ante los servicios sanitarios por dificultades de accesibilidad, falta de 
información y las necesidades específicas que se derivan de su particular situación. Para ello hemos planteado los siguientes objetivos específicos:

Objetivo1. Describir cómo perciben la asistencia sanitaria durante el desarrollo del embarazo y parto las mujeres murcianas con discapacidad.

a. ¿Perciben, las mujeres con discapacidad, barreras físicas que le impidan disfrutar de la asistencia sanitaria como cualquier mujer?

b. ¿Perciben falta de información durante el proceso de embarazo y parto?

c. ¿Disponen de poca formación los profesionales sanitarios en el tema de la discapacidad, desde el punto de vista de la asistencia percibida?

d. ¿Advierten más medicalizados los embarazos y partos de las mujeres con discapacidad?

e. ¿Se muestran, los profesionales sanitarios, ante las mujeres con discapacidad, influenciados por estereotipos sociales?

Objetivo 2.Valorar la percepción que, desde las asociaciones de discapacitados de la Región de Murcia, se tiene del embarazo y parto en las mujeres con discapacidad.

Objetivo 3. Identificar características clínicas de embarazos y partos en mujeres con discapacidad física. Durante el período comprendido entre 2005-2009, en un hospital público de la Región de Murcia.

\section{MATERIAL Y MÉTODO}

Para poder profundizar en algunos aspectos de la realidad vital de las mujeres con discapacidad de la región de Murcia, durante su embarazo y parto hemos utilizado técnicas de investigación cualitativa y la perspectiva asistencial-sanitaria, intentando conocer cómo han percibido el trato asistencial durante el proceso de embarazo y parto, las vivencias y sus significados. Además, hemos realizado una entrevista a los directores de las distintas asociaciones de discapacitados en Murcia, con preguntas abiertas para valorar la percepción que tienen desde la asociación de la maternidad en sus asociadas. Para este estudio, también se han utilizado técnicas cualitativas.

Finalmente, hemos pensado adecuado apoyar el estudio describiendo la asistencia sanitaria con datos clínicos y, en consecuencia, hemos utilizado técnicas de investigación cuantitativa para poner de manifiesto algunas de las características clínicas de embarazos y partos en mujeres con discapacidad física.

\section{Población, muestra y entorno}

\section{Población:}

En el estudio cualitativo (objetivos 1 y 2), la población comprende a las mujeres con discapacidad física de la Región de Murcia. La vía de acceso a ellas ha sido a través de las diferentes asociaciones de discapacitados que existen en la Región, que constituyen la población del objetivo 2.

La población de nuestro estudio se reparte entre las nueve áreas de salud, en las que se divide la Región de Murcia, para organizar su asistencia sanitaria. Que son las que enumeramos en la Tabla I. 
Tabla I. Áreas de Salud de la Región de Murcia.

\begin{tabular}{|l|l|l|}
\hline \multicolumn{1}{|c|}{ Área } & \multicolumn{1}{|c|}{ Denominación } & \multicolumn{1}{c|}{ Hospital de referencia } \\
\hline I & Murcia/Oeste & Hospital Universitario "Virgen de la Arrixaca" \\
\hline II & Cartagena & Hospital Universitario "Santa María del Rosell”. \\
\hline III & Lorca & Hospital "Rafael Méndez" \\
\hline IV & Noroeste & Hospital Comarcal del Noroeste \\
\hline V & Altiplano & Hospital "Virgen del Castillo" \\
\hline VI & Vega Media del Segura & Hospital Universitario "Morales Meseguer" \\
\hline VII & Murcia/Este & Hospital General Universitario "Reina Sofía" \\
\hline VIII & Mar Menor & Hospital "Los Arcos" \\
\hline IX & Vega Alta del Segura & Hospital "Vega del Río Segura" \\
\hline
\end{tabular}

Para el estudio cuantitativo (objetivo 3), hemos elegido un hospital público de la Región de Murcia.

\section{Muestra:}

La muestra de la parte cualitativa del estudio, está formada por cinco mujeres con diferentes discapacidades físicas (acondroplasia, esclerosis múltiple, arreflexia general, hemiplejía derecha, fibrosis quística), que han sido madres durante el periodo comprendido entre 20052009. La selección e incorporación de las mujeres a la muestra la hemos realizado siguiendo el procedimiento denominado "muestreo no probabilístico por bola de nieve" ${ }^{35}$, debido a la dificultad para acceder a ellas, por falta de existencia de registros en las distintas asociaciones analizadas. El número total de participantes no se obtuvo sobre la base de un valor numérico predefinido, sino que este fue formándose hasta que se alcanzó la saturación de los datos, y no se obtuvo nueva información ${ }^{36}$

La muestra del objetivo 2 está formada por 2 de las 17 asociaciones consultadas, y enumeradas anteriormente; se ha cuidado que todas sean asociaciones con discapacidad de tipo físico, descartando aquellas cuyos asociados presentan discapacidad mental, intelectuales o sensoriales por no ser objeto de nuestro estudio. La selección se obtuvo dirigiéndonos a la sede de la Federación de Asociaciones Murcianas de Personas con Discapacidad Física y/u Orgánica (FANDIF), desde donde se nos proporcionaron las direcciones del resto de asociaciones.

Para recoger la muestra del objetivo 3, la parte cuantitativa del estudio, analizamos de forma retrospectiva las historias clínicas de las mujeres con discapacidad que habían sido madres durante los últimos cinco años en el hospital. Para ello, solicitamos información clínica en el Servicio de Codificación del mencionado hospital, encontrando la dificultad añadida de que la discapacidad no viene recogida en la codificación de las historias clínicas, por no ser un diagnóstico médico. Para obtener una muestra adecuada se buscaron códigos de patologías que pudieran causar discapacidad y se casaron con códigos de embarazo y parto. Tras sucesivas visitas a la mencionada unidad, finalmente se logró seleccionar un total de 3.335 casos de 186.394 registros desde el año 2005 hasta el 2009. En ellos se incluyen los siguientes Grupos relacionados con Diagnósticos (GRDs): 370-377 (Referentes a embarazo y parto, cesárea, con y sin complicaciones, que vienen recogidos en la Clasificación Internacional de Enfermedades ${ }^{34}$ ) asociados a los siguientes diagnósticos principales 0 secundarios: 277 Fibrosis quística, 340 esclerosis múltiple, 268 raquitismo, 741 espina 
bífida, 756 condrodistrofia, 754 luxación congénita de caderas, 720 espondilitis anquilosante, 714 artritis reumatoide, 745 transposición de grandes vasos, 747 aneurisma de aorta congénitas. Posteriormente, la muestra se agrupó en tres grandes diagnósticos que fueron: 648.91 otras enfermedades clasificables bajo otros conceptos (que incluye las cinco primeras), 648.71 trastornos de huesos y articulaciones de la espalda, pelvis y miembros inferiores (que incluye las tres siguientes) y 648.51 trastornos cardiovasculares congénitos (los dos últimos).

\section{Entorno:}

La muestra fue recogida en un entorno natural, es decir en una situación no controlada del entorno, en la vida real de las mujeres con discapacidad. La muestra fue recogida durante los meses de Abril a Junio de 2010.

\section{Criterios de exclusión.}

La definición del instrumento de ratificación de la convención de los derechos de las personas con discapacidad incluyen a aquellas que tengan deficiencias físicas, mentales, intelectuales o sensoriales a largo plazo que, al interactuar con diversas barreras, puedan impedir su participación plena y efectiva en la sociedad, en igualdad de condiciones con las demás.

Para nuestro estudio, hemos excluido a las personas que presentan deficiencias mentales, intelectuales o sensoriales. Centramos el estudio en las deficiencias físicas que pudieran presentar problemas con la gestación y el parto.

\section{Instrumentos.}

\section{Estudio cualitativo.}

En las entrevistas, hemos utilizado un cuestionario de satisfacción de las mujeres con el seguimiento del embarazo ${ }^{37}$, con preguntas semiestructuradas y abiertas, y lo hemos adaptado a la discapacidad. En reconocimiento a la contribución de los participantes y para validar los datos, se les ha informado sobre los resultados de la investigación

\section{Estudio cuantitativo}

Tras elaborar un listado de las patologías que podían producir discapacidad física, utilizamos la base de datos del servicio de codificación del hospital para seleccionar las historias, posteriormente utilizamos una hoja de cálculo para agrupar y analizar los datos.

\section{Procedimiento para la recopilación de datos.}

\section{Estudio cualitativo.}

Para la recopilación de datos, las entrevistas, se grabaron en audio para su posterior trascripción literal y análisis. La duración de las entrevistas fue de $30 \mathrm{~min}$. Después de una lectura en profundidad, se codificaron los datos identificando las palabras, frases o temas que pudiesen tener un significado destacable en relación a los objetivos de estudio. A continuación, se hizo la definición operacional de éstos. Como segundo paso se reagrupó bajo una misma etiqueta, aquellos códigos que compartían significado lo que nos guió a la 
identificación de los temas esenciales del trabajo que destacamos en el apartado de resultados.

En la realización de las encuestas, nos comprometemos por escrito al anonimato y confidencialidad de los datos. Se explicó a las participantes seleccionadas los objetivos generales de la investigación y se les informó que la recogida de datos sería grabada. De igual forma, se les garantizó el anonimato, la confidencialidad de los datos obtenidos (todas las participantes fueron inicialmente codificadas lo que no permitía su identificación durante el análisis y los datos originales son conservados en una base de datos a la que sólo tiene acceso la investigadora principal) y la libertad de abandonar el estudio en cualquier momento. Se requirió consentimiento formal a las participantes.

\section{Estudio cuantitativo}

En el servicio de Codificación del hospital, solicitamos tener acceso a las Historias Clínicas de mujeres con una patología determinada, se nos facilitó un listado de las mencionadas historias agrupadas por códigos, mostrados en el apartado de resultados. Para la obtención de Historias clínicas por parte del hospital, se firmó un documento de confidencialidad.

\section{Diseño del estudio.}

\section{Estudio cualitativo.}

Para describir cómo perciben la asistencia sanitaria las mujeres con discapacidad durante el desarrollo del embarazo y el parto, utilizamos la metodología cualitativa, realizando un estudio fenomenológico, porque según De la Cuesta $^{38}$, nos permite comprender una experiencia tal como es vivida por la persona. La fenomenología no mide un fenómeno, sino que lo describe e interpreta para que se conozca de una manera más profunda. El propósito de la fenomenología es estudiar y describir la esencia de la experiencia humana vivida. Por todo ello, utilizamos este tipo de investigación para conseguir el propósito de identificar y conocer en profundidad la percepción, visión y experiencia que tienen las mujeres con discapacidad sobre la asistencia sanitaria recibida durante su proceso de embarazo y parto.

\section{Estudio cuantitativo.}

Para identificar características clínicas de embarazos y partos en mujeres con discapacidad física en el hospital, realizamos un estudio retrospectivo, puesto que queríamos valorar el periodo comprendido entre 2005-2009, utilizamos para ello la metodología cuantitativa, realizando un análisis descriptivo de los procesos encontrados.

El problema de validez interna del estudio, viene de la codificación de los datos en el hospital, pues la discapacidad no viene codificada, y por tanto, hemos solicitado historias de mujeres con un determinado tipo enfermedad, que creemos que puede producir una discapacidad, pero sabemos que una misma enfermedad puede producir en personas distintas, diferentes grados de discapacidad.

Para obtener el consentimiento informado, en el estudio cualitativo, antes de la realización de las entrevistas, se entregaba una hoja informando a los participantes sobre las características del trabajo de investigación, sus riesgos y beneficios, confidencialidad y derechos. Para el estudio cuantitativo, se ha necesitado realizar una solicitud para tener acceso a las historias clínicas del hospital, junto con la firma de un documento en el que se asegura la confidencialidad en el uso de los datos solicitados. 


\section{RESULTADOS}

\section{Resultados objetivo 1: "describir cómo perciben la asistencia sanitaria las mujeres con discapacidad durante el desarrollo del embarazo y parto".}

Los resultados de las entrevistas se presentan a continuación:

\section{Datos sociodemográficos. (Tabla II)}

Las cinco participantes fueron mujeres, cuatro de ellas de nacionalidad española, y una marroquí. En el momento de la entrevista, las mujeres tenían entre 30-45 años y sus hijos entre 0 y 14 años, tres mujeres tenían dos hijos y una, un hijo. Todas estaban casadas o con pareja estable, todas disponían de apoyo familiar y/o de la pareja. El nivel educativo de cuatro de ellas eran estudios primarios, una tenía estudios superiores. Dos trabajaban fuera de casa.

Las patologías que presentaban eran: acondroplasia, esclerosis múltiple, arreflexia global polineuropatía, hemiplejía derecha y fibrosis quística. El grado de minusvalía oscilaba entre $45-75 \%$

Todas recibieron la noticia del embarazo con alegría. Eran embarazos buscados y deseados.

Tabla II. Datos sociodemográficos de las participantes. E1-E5: Mujeres entrevistadas.

\begin{tabular}{|c|c|c|c|c|c|c|c|c|c|}
\hline & EDAD & $\begin{array}{l}\text { Nivel } \\
\text { Educativo }\end{array}$ & $\begin{array}{l}\text { Situación } \\
\text { Laboral }\end{array}$ & $\begin{array}{l}\text { Estado } \\
\text { Civil }\end{array}$ & $\begin{array}{l}\mathrm{N}^{\circ} \\
\text { Hijos }\end{array}$ & $\begin{array}{l}\text { Lugar de } \\
\text { Nacimiento }\end{array}$ & Deficiencia & $\begin{array}{l}\text { Grado de } \\
\text { Minusvalía. }\end{array}$ & $\begin{array}{l}\text { Apoyo al } \\
\text { embarazo }\end{array}$ \\
\hline E1 & 41 & $\begin{array}{l}\text { ESTUDIOS } \\
\text { PRIMARIOS }\end{array}$ & TRABAJA & $\begin{array}{l}\text { PAREJA } \\
\text { ESTABLE }\end{array}$ & 2 & MURCIA & $\begin{array}{l}\text { ACONDRO- } \\
\text { PLASIA }\end{array}$ & 65 & $\begin{array}{l}\text { SI } \\
\text { PAREJA }\end{array}$ \\
\hline E2 & 32 & $\begin{array}{l}\text { ESTUDIOS } \\
\text { PRIMARIOS }\end{array}$ & $\begin{array}{l}\text { NO } \\
\text { TRABAJA }\end{array}$ & CASADA & 2 & MURCIA & $\begin{array}{l}\text { ESCEROSIS } \\
\text { MULTIPLE }\end{array}$ & 66 & $\begin{array}{l}\text { SI PAR } \\
\text { Y FAM. }\end{array}$ \\
\hline E3 & 38 & $\begin{array}{l}\text { ESTUDIOS } \\
\text { PRIMARIOS }\end{array}$ & $\begin{array}{l}\text { NO } \\
\text { TRABAJA }\end{array}$ & CASADA & 2 & SEVILLA & $\begin{array}{l}\text { ARREFLEXIA } \\
\text { GENERAL }\end{array}$ & 45 & $\begin{array}{l}\text { SI PAR } \\
\text { Y FAM. }\end{array}$ \\
\hline E4 & 32 & $\begin{array}{l}\text { ESTUDIOS } \\
\text { PRIMARIOS }\end{array}$ & $\begin{array}{l}\text { NO } \\
\text { TRABAJA }\end{array}$ & CASADA & 1 & MARRUECOS & $\begin{array}{l}\text { HEMIPARESIA } \\
\text { DERECHA }\end{array}$ & 65 & $\begin{array}{l}\text { SI PAR } \\
\text { Y FAM. }\end{array}$ \\
\hline E5 & 33 & $\begin{array}{l}\text { ESTUDIOS } \\
\text { SUPERIORES }\end{array}$ & TRABAJA & CASADA & 1 & MURCIA & $\begin{array}{l}\text { FIBROSIS } \\
\text { QUISTICA }\end{array}$ & 65 & $\begin{array}{l}\text { SI.PAR } \\
\text { Y FAM. }\end{array}$ \\
\hline
\end{tabular}

¿Perciben, las mujeres con discapacidad, barreras físicas, que le impidan disfrutar de la asistencia sanitaria como cualquier mujer?

Las preguntas 16 y 17 de nuestro cuestionario, se dirigían específicamente para valorar esta cuestión. (Tabla III). Tres de las cinco entrevistadas, se quejaban de que las camillas obstétricas son demasiado altas, que no lograban acceder a ellas sin ayuda, normalmente iban a la consulta acompañadas y eran sus parejas quienes se encargaban de ayudarlas.

E1: "Tuve que soportar cuatro personas mirándome, y yo ahí desnuda haciendo malabarismos para poder subirme al potro con la barriga de ocho meses...hasta que la enfermera se decidió a ayudarme" 
E3: "En el hospital, dejaron fuera a mi pareja y ya no tenía a nadie que me ayudara a subirme a la cama, y yo no llegaba..."

Tres de las entrevistadas refieren que el personal sanitario no les ayudaba. Una de ellas refiere que en Primaria le ayudaba la matrona.

\section{¿Perciben falta de información durante el proceso de embarazo y parto?}

Las preguntas $22-26$ y 28 del cuestionario estaban relacionadas con la información recibida durante las consultas médicas, los resultados.

El ser siempre atendidas por el mismo médico, lo refieren como importante para un mejor seguimiento y consideran que así no tienen que contar "su historia" una y otra vez.

Tres de las encuestadas piensa que el trato en el hospital se podría mejorar y no están satisfechas. Las dos atendidas en Atención Primaria se muestran satisfechas con el trato recibido.

De las que fueron atendidas en el hospital, dos no se sintieron escuchadas por el profesional que se encargaba de su embarazo, tres de ellas no sentían que se tomaran interés por ellas y por su discapacidad. Además, no recibieron educación sanitaria, porque no se la ofrecieron. Excepto una de ellas que no se vio en la necesidad de preguntar, porque todo el embarazo se desarrolló con normalidad y estaba bien informada por sus propios medios.

Las que fueron atendidas en Atención Primaria se muestran más satisfechas y se resolvían sus dudas, se les ofreció educación sanitaria, una de ellas no la recibió por dificultad para acceder al centro de salud, pero se la ofrecieron.

¿Disponen de poca formación los profesionales sanitarios en el tema de la discapacidad, desde el punto de vista de la asistencia percibida?

La pregunta 27 del cuestionario hace referencia directa a esta cuestión. Tres de las encuestadas creen que el profesional que les atendió sí estaba formado para abordar su discapacidad en relación con el embarazo. El resto, asegura que no, la impresión que recibieron fue que se les evitaba el tema, remitiéndole a su médico habitual.

E2: "Sólo le importaba mi embarazo, cuando le preguntaba algo relacionado con mi enfermedad, me remitía a mi médico habitual sin resolverme las dudas"

Las que fueron atendidas en el hospital no consideran que las explicaciones fueran adecuadas y no se resolvían sus dudas en la consulta, ya que no les ofrecían la confianza para preguntar libremente sus dudas por lo que no creen que el profesional estuviese preparado para abordar su discapacidad 
Tabla III. Asistencia durante el embarazo. E1-E5: Mujeres entrevistadas

\begin{tabular}{|l|l|l|l|l|l|l|l|}
\hline & CONTROL & $\begin{array}{l}\text { DIFICULTAD } \\
1^{2} \text { CITA }\end{array}$ & $\begin{array}{l}\text { CONSULTA } \\
\text { ADECUADA }\end{array}$ & MISMO MEDICO & $\begin{array}{l}\text { No PRUEBAS } \\
\text { SUFICIENTES }\end{array}$ & TRATO PERSONAL & $\begin{array}{l}\text { ESCUCH } \\
\text { ADA }\end{array}$ \\
\hline E1 & HOSPITAL & NO & NO & SI & SI & PODRIA MEJORAR & NO \\
\hline E2 & $\begin{array}{l}\text { HOSPITALY } \\
\text { PRIVADA }\end{array}$ & SI & SI & SO & $\begin{array}{l}\text { MENOS DE LAS } \\
\text { NECESARIAS. }\end{array}$ & $\begin{array}{l}\text { PODRIA } \\
\text { MEIORAR }\end{array}$ & NO \\
\hline E3 & $\begin{array}{l}\text { ATENCION } \\
\text { PRIMARIA }\end{array}$ & NO & NO & SI & BIEN & SI \\
\hline E4 & $\begin{array}{l}\text { ATENCION } \\
\text { PRIMARIA }\end{array}$ & SI & SI & SI & SI & BIEN & SI \\
\hline E5 & HOSPITAL & NO & SI & SI & SI & BIEN \\
\hline ADECUADAS & PROFESIONAL & $\begin{array}{l}\text { RESOLUCION } \\
\text { DUDAS }\end{array}$ & CONFIANZA & $\begin{array}{l}\text { PREPARACION } \\
\text { PROFESIONAL } \\
\text { DISCAPACIDAD }\end{array}$ & EDUCACION \\
SANITARIA & \\
\hline E1 & NO & NO & NO & NO & NO & NO / NO OFRECIERON \\
\hline E2 & NO & NO & NO & NO & NO & NO/ NO OFRECIERON \\
\hline E3 & SI & NO & SI & SI & SI & NO/ DIFICULTAD ACCESO \\
\hline E4 & SI & SI & SI & SI & SI & SI/ MATRONA \\
\hline E5 & SI & SI & SI & SI & SI & SI/MATRONA PRIVADA \\
\hline
\end{tabular}

¿Advierten más medicalizados los embarazos y partos, las mujeres con discapacidad?

La pregunta 20 del cuestionario y las preguntas referidas a la asistencia durante el parto, responden a esta cuestión. (Tabla III)

Cuatro creen que se les realizó el número de pruebas suficientes, una de ellas expresaba que fueron menos de las que necesitaba, por lo que recurrió a la asistencia privada.

Tres de las cinco encuestadas finalizaron el proceso de embarazo con una cesárea programada por su médico. Las otras dos mujeres tuvieron un parto vaginal, una de ellas inducido y el otro espontáneo.

E2: “...Me fui a la privada porque yo quería un parto vaginal y en el hospital me dijeron que sería una cesárea programada..." (En el hospital privado tuvo un parto vaginal sin complicaciones).

Es de destacar, en los resultados, que la totalidad de las cesáreas programadas de nuestras participantes, no fueron vistas previamente en la consulta de preanestesia.

E5: "No pasé la preanestesia porque se les olvidó". 
E1: "En el antequirófano tuve que discutir con los anestesistas, pues querían ponerme una epidural y yo sabía que eso no era adecuado para mí por mi patología, no me creyeron y fueron a consultarlo por Internet..."

Los resultados de los cinco partos fueron niños sanos, dos de ellos fueron ingresados en neonatología para observación, con peso adecuado a su edad gestacional. Ninguna de ellas tuvo problemas durante el posparto y cuatro pudieron dar lactancia materna a sus hijos, tal como deseaban. (Tabla IV)

¿Se muestran, los profesionales sanitarios, ante las mujeres con discapacidad, influenciados por estereotipos sociales?

E1: “...Tras la cesárea, escuché a los ginecólogos comentar mi intervención, las diferencias encontradas en mi interior debido a mi enfermedad, y mofarse de que no quisiera la ligadura..."

Además de estas cuestiones, obtuvimos un resultado que merece ser destacado, es el sentimiento de desprotección que refieren nuestras entrevistadas, ante la asistencia sanitaria percibida en el hospital.

E1: "Cuando pienso en el hospital, me aterrorizo, me siento desprotegida en ese medio..."

E2: "Me tuve que ir a "la privada" porque en el hospital no me daban opción a elegir, tenía que ser cesárea, y yo quería un parto vaginal, no me sentía respetada".

Los resultados recogen falta de sensibilidad en el trato, falta de intimidad y finalmente, creen que el trato profesional se podría mejorar.

Tabla IV. Asistencia durante el parto. E1-E5: Mujeres entrevistadas

\begin{tabular}{|l|l|l|l|l|l|l|}
\hline & TIPO PARTO & INGRESO RN & PESO RN & $\begin{array}{l}\text { EDAD } \\
\text { GEST }\end{array}$ & $\begin{array}{l}\text { DIFICULTAD } \\
\text { POSPARTO }\end{array}$ & $\begin{array}{l}\text { LACTANCIA } \\
\text { MATERNA }\end{array}$ \\
\hline E1 & $\begin{array}{l}\text { CESAREA } \\
\text { PROGRAMADA }\end{array}$ & SI & $i ?$ & 37 & NO & NO \\
\hline E2 & $\begin{array}{l}\text { VAGINAL } \\
\text { INDUCIDO }\end{array}$ & NO & 2730 & $36+6$ & NO & SI \\
\hline E3 & $\begin{array}{l}\text { CESAREA } \\
\text { PROGRAMADA }\end{array}$ & NO & 3190 & $40+6$ & NO & SI \\
\hline E4 & $\begin{array}{l}\text { VAGINAL } \\
\text { ESPONTANEO }\end{array}$ & NO & 3560 & $41+2$ & NO & SI \\
\hline E5 & $\begin{array}{l}\text { CESAREA } \\
\text { PROGRAMADA }\end{array}$ & SI & 3680 & 38 & NO & \\
\hline
\end{tabular}


2 Resultados objetivo 2: "valorar la percepción que, desde las asociaciones de discapacitados de la Región de Murcia, se tiene del embarazo y parto en las mujeres con discapacidad".

Para realizar este estudio, utilizamos un cuestionario de elaboración propia con preguntas abiertas.

Los resultados de las encuestas realizadas a las asociaciones de discapacitados de la Región de Murcia, muestran un índice de no respuesta muy alto, ya que tan solo responden dos de las diecisiete asociaciones consultadas. (Tabla V)

Destacamos que las mujeres asociadas acuden a la asociación buscando información acerca del embarazo y parto.

Tabla V. Entrevista asociaciones de discapacitados de la Región de Murcia.

\begin{tabular}{|l|l|l|l|l|l|l|}
\hline ASOCIACION & No SOCIOS & MUJERES & $\begin{array}{l}\text { MADRES } \\
\text { /EMBARAZ. }\end{array}$ & $\begin{array}{l}\text { RECOGEN } \\
\text { PROBLEMÁTICA } \\
\text { SALUD } \\
\text { REPRODUCTIVA }\end{array}$ & $\begin{array}{l}\text { PROBLEMAS } \\
\text { ENFERMEDAD } \\
\text { /GESTACION }\end{array}$ & $\begin{array}{l}\text { ACUDEN A LA } \\
\text { ASOCIACION } \\
\text { BUSCANDO } \\
\text { INFORMACION }\end{array}$ \\
\hline $\begin{array}{l}\text { FIBROSIS } \\
\text { QUISTICA }\end{array}$ & 125 & 46 & 6 & SI & PULMONARES & SI \\
\hline $\begin{array}{l}\text { ESCLEROSIS } \\
\text { MULTIPLE }\end{array}$ & 254 & 153 & $\begin{array}{l}\text { LO } \\
\text { DESCONOCEN }\end{array}$ & NO & $\begin{array}{l}\text { EL EMBARAZO } \\
\text { NO ALTERA } \\
\text { EL CURSO DE } \\
\text { LA } \\
\text { ENFERMEDAD. } \\
\text { / USO DE } \\
\text { FARMACOS }\end{array}$ & SI \\
\hline
\end{tabular}

También quisimos constatar el género de los directores de las asociaciones de discapacitados de la Región de Murcia, con el fin de valorar su repercusión a la hora de hacerse eco de los problemas de salud reproductiva en la mujer con discapacidad. El resultado fue de 19 asociaciones consultadas, 10 directores eran mujeres y el resto hombres, por tanto no existen diferencias de género en la dirección de las distintas asociaciones de discapacitados de la Región de Murcia.

3 Resultados objetivo 3: "Identificación de las características clínicas de embarazos y partos en mujeres con discapacidad física, analizados en el hospital, durante el periodo comprendido entre 2005-2009".

Se obtuvieron un total de 3374 casos asistidos en el hospital, en los que se valoró la terminación del proceso de embarazo, obteniendo los siguientes resultados (Figura 1): 1652 partos sin complicaciones, 1094 partos con complicaciones, 494 cesáreas sin complicaciones y 134 cesáreas con complicaciones. 
Figura 1. Finalización de procesos de embarazo en mujeres con discapacidad en el hospital. Fuente: Servicio de Codificación del hospital.

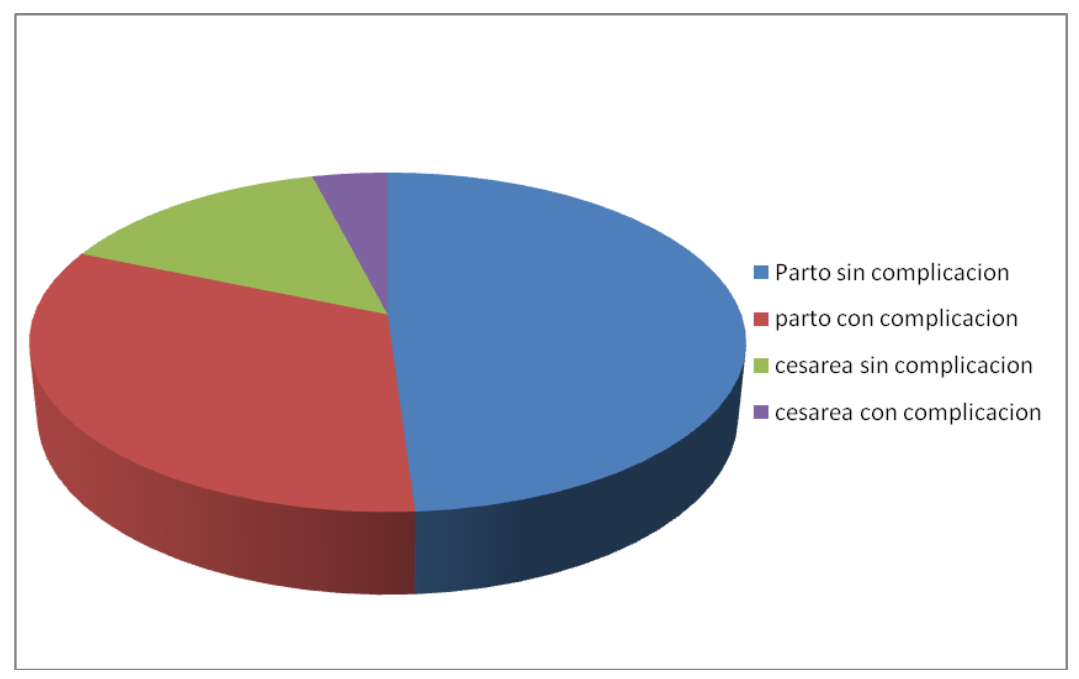

El número total de cesáreas en mujeres con discapacidad, durante el periodo 2005-2009, fue de un $18,6 \%$. Al comparar estos resultados con el total de partos y cesáreas atendidos en el hospital (población con o sin discapacidad), nos encontramos que el número de cesáreas en las mujeres con discapacidad fue similar a la de las mujeres sin discapacidad (Tabla VI).

Tabla VI. Fuente: Servicio de Administración del hospital.

\begin{tabular}{|l|l|l|l|}
\hline AÑO & TOTAL & PARTOS & CESAREAS \\
\hline 2007 & 8249 & 6285 & $1964(23.8 \%)$ \\
\hline 2008 & 8457 & 7062 & $1395(16.4 \%)$ \\
\hline 2009 & 8230 & 6285 & $1470(17.8 \%)$ \\
\hline
\end{tabular}

\section{DISCUSIÓN Y CONCLUSIÓN}

\section{Discusión}

Vamos a enumerar los diferentes objetivos específicos, para discutir cada uno de ellos por separado:

1. Describir cómo perciben la asistencia sanitaria las mujeres con discapacidad durante el desarrollo del embarazo y parto.

a. ¿Perciben, las mujeres con discapacidad, barreras físicas, que le impidan disfrutar de la asistencia sanitaria como cualquier mujer?

La estructura física, los recursos materiales y humanos, de los que disponen las organizaciones sanitarias, generan condiciones que en ocasiones son poco favorables para ejercer un adecuado cuidado de lo íntimo y del mantenimiento de la intimidad ${ }^{41}$. Pero no debemos olvidar que, a pesar de tener a nuestro alcance muchos recursos, (biombos, puertas, cortinas) algunos profesionales no los utilizan, por mecanización en sus conductas, dando lugar a una disminución en la calidad asistencial, no consiguiendo el nivel óptimo por falta del "detalle fino", ya que la calidad científico-técnica de los cuidados si es reconocida por la mujer. ${ }^{42}$ 
Nuestras entrevistadas coinciden en este punto con los autores mencionados, se quejan de camas obstétricas demasiado altas y de difícil acceso y también de falta de sensibilidad de los profesionales a la hora de paliar las barreras físicas que le impidan disfrutar de una óptima asistencia.

b. ¿Perciben falta de información durante el proceso de embarazo y parto?

Existen diferencias significativas entre las mujeres controladas desde el ámbito de la Atención Primaria, donde son atendidas por un equipo multidisciplinar (obstetra, matrona y médico de familia) y las que han sido seguidas desde Atención Especializada, es decir, por el obstetra del hospital, privando a las gestantes de la atención regular de la matrona, fuente importante de información y cuidados para la salud.

En general, las mujeres de nuestro estudio refieren haber recibido poca información sanitaria en la consulta médica, teniendo que recurrir a buscarla por su cuenta en caso de necesitarla. En este punto coincidimos con los resultados de diferentes estudios. ${ }^{25-45}$

c. ¿Disponen de poca formación los profesionales sanitarios en el tema de la discapacidad, desde el punto de vista de la asistencia percibida algunos?

En un estudio realizado a nivel nacional en Houston, concluyen que el personal sanitario que tiene que atender a la mujer durante el embarazo y el parto, tienen poca experiencia en el manejo de la discapacidad, debido a que médicos de atención primaria, obstetras, ginecólogos y matronas, en general, reciben muy poca o ninguna formación en el efecto de la discapacidad en la salud reproductiva de las mujeres. 11 En nuestro estudio, la poca formación de nuestros profesionales, es percibida por tres de las cinco encuestadas y así lo reflejan en la entrevista.

d. ¿Advierten más medicalizados los embarazos y partos de las mujeres con discapacidad?

La OMS insiste en la necesidad de reducir el número de cesáreas ${ }^{43}$, la valoración individual de las gestantes contribuiría en gran medida a disminuir el número de cesáreas programadas a causa de la discapacidad física materna. La cifra de cesáreas en torno a $18.6 \%$ en mujeres con discapacidad, se aleja de las recomendaciones de la OMS, que estaría en torno al $10 \%$, pero es parecida a las cifras que habitualmente se vienen manejando en el hospital, al resto de la población. Por ello, no tenemos motivos para pensar que la presencia de discapacidad aumente la probabilidad de sufrir una cesárea en el momento del parto en el hospital. Por otro lado, cuatro de nuestras cinco entrevistadas estaban programadas para finalizar su embarazo con cesáreas. Una de ellas, decidió ser atendida de forma privada porque deseaba un parto vaginal, y pudo realizarse sin ninguna complicación. Esto nos puede indicar que en dicho Hospital se programan cesáreas cuando existe un mínimo riesgo de complicación, sea por motivo de discapacidad u otro motivo. Por ello, no encontramos diferencias entre nuestras mujeres y el resto sin discapacidad.

La medicalización también afecta al servicio de anestesia, según el Manual de Procedimientos de la Consulta de Preanestesia del Consorcio Hospital Universitario de Valencia ${ }^{44}$, cualquier paciente programado para cirugía electiva debe ser evaluado por el anestesiólogo en el periodo preoperatorio, con el fin de reducir al máximo la 
morbilidad asociada con la cirugía y la administración de la anestesia, optimizar el estado de salud del paciente antes de la cirugía y planear el manejo perioperatorio más adecuado, seleccionar y optimizar recursos, detección de pacientes de alto riesgo, informar y obtener el consentimiento y aliviar la ansiedad. Así, nos surgen dos preguntas durante el desarrollo de este estudio: ¿se le da la importancia médica que tiene a la intervención quirúrgica de la cesárea programada? ¿Es normal que ninguna de las cesáreas programadas de nuestro estudio pasara por una consulta de preanestesia?

e. ¿Se muestran, los profesionales sanitarios, ante las mujeres con discapacidad, influenciados por estereotipos sociales?

El hecho de atender los embarazos de las mujeres con discapacidad como de alto riesgo, supone aceptar un estereotipo que es "discapacidad igual a complicaciones en el embarazo" ${ }^{45}$ En nuestro estudio, no todas se han atendido como embarazos de alto riesgo, dos de ellas han sido atendidas en Atención Primaria, lo que nos hace suponer que la asistencia sanitaria, ha sido individualizada.

Encontramos que los resultados de nuestras entrevistas, que valoran la influencia de los profesionales sanitarios por estereotipos sociales, no son aplicables, en este caso, más bien creemos que obedecen a una falta de respeto a la intimidad de la persona atendida.

E1: “...Tras la cesárea, escuché a los ginecólogos comentar mi intervención, las diferencias encontradas en mi interior debido a mi enfermedad, y mofarse de que no quisiera la ligadura..."

Se puede constatar que el respeto a la intimidad, que es un derecho fundamental ligado a la dignidad de la persona, y la obligación de guardar la confidencialidad que afecta a todo el personal que trabaja en el hospital, no sólo en la recogida de la información, sino también en el uso que se haga de la misma, son obviados en algunos momentos. A menudo, se comentan temas con otros profesionales. Se deberían evitar estas situaciones que suponen una de las formas más frecuentes e inconscientes de agredir la confidencialidad, coincidiendo con la literatura revisada. ${ }^{42}$

2. Valorar la percepción que, desde las asociaciones de discapacitados de la Región de Murcia, se tiene del embarazo y parto en las mujeres con discapacidad.

La labor de las Asociaciones es muy importante como fuente de información, las mujeres acuden a ellas en busca de la información que necesitan, en ellas se le proporciona y se les deriva a los profesionales médicos que tienen de referencia. En nuestro estudio, el alto índice de no respuesta, puede indicar que no han recogido esta problemática entre sus asociadas Además, no todas disponen de datos estadísticos respecto a los miembros que las forman, lo que sería recomendable para poder conocer mejor los problemas derivados de la discapacidad e intentar prevenirlos o solucionarlos .

3. Identificar características clínicas de embarazos y partos en mujeres con discapacidad física. Durante el período comprendido entre 2005-2009, en el hospital.

De los resultados anteriores, nos llamó la atención dos aspectos, en primer lugar, el número de partos con complicación. En un segundo análisis, buscamos la causa del número de partos con complicación tan elevado, y desde el servicio de codificación se nos informó, que 
consideran complicación a multitud de situaciones, que en la práctica clínica no son tal, (por ejemplo una circular de cordón al cuello, un cordón corto, una primípara añosa, retardo en alumbrar, etc. ${ }^{34}$

En segundo lugar, el número de cesáreas (considerado un índice de intervencionismo médico) cifrado en un $18.6 \%$, es ligeramente superior a la tendencia normal. Somos conscientes de que nuestros datos engloban a los cinco últimos años, y que el índice anual de cesáreas varía (no se nos han facilitado datos anteriores a 2007 en el servicio de Administración del hospital respecto al índice de cesáreas). En cualquier caso podemos deducir que, en general, en el hospital se hacen cesáreas programadas para evitar complicaciones en el niño o madre. Esto implica que sería necesario un estudio más profundo y detallado para saber si realmente están justificadas las cesáreas realizadas a las pacientes con discapacidad.

\section{CONCLUSIONES}

Se deduce de la investigación, que las mujeres con discapacidad que han sido atendidas, durante el embarazo en Atención Primaria, se sienten más satisfechas con el trato recibido; por otro lado las que han sido controladas en Atención Especializada, no solo no se sienten satisfechas, sino que además se observan carencias en cuanto a información y educación sanitaria recibida.

Según los datos del estudio no existen datos suficientes para afirmar que se realicen mayor número de cesáreas, debidas a la discapacidad de la mujer, que al resto de la población.

Sin embargo, las mujeres con discapacidad perciben una falta de sensibilidad en el trato de los profesionales sanitarios y una falta de respeto a su intimidad, y por otro lado, las Asociaciones de discapacitados de la Región, no han recogido esta problemática entre los objetivos de su asociación.

\section{AGRADECIMIENTOS}

Nuestro más profundo agradecimiento a todas las personas que de diversas formas han colaborado en la elaboración de este trabajo de investigación, bien de una forma concreta y práctica o con su apoyo moral.

A las distintas asociaciones de discapacitados de la Región de Murcia, que tan generosamente nos han ofrecido su tiempo y dedicación para poder realizar esta investigación.

Al Servicio de Codificación del Hospital, a su responsable, Román Picazo y en especial a Marisa Argemi, sin los cuales no dispondría de datos clínicos, gracias a su tesón y esfuerzo desinteresado.

Al servicio de administración del hospital maternal.

Y especialmente a las mujeres de nuestra investigación, gracias a las cuales ha sido posible realizar esta investigación y nos han permitido profundizar un poco más en el tema de la discapacidad, aportándonos sus vivencias, con la única finalidad de mejorar la práctica clínica de la asistencia al embarazo y parto de las mismas. 


\section{BIBLIOGRAFÍA}

1. Aguado, AL."Historia de las deficiencias". Madrid: Libre Editorial, Fundación ONCE; 1995.

2. UN enable.org, Historia de la discapacidad y las Naciones Unidas. Naciones Unidas; 2010. Disponible en:

http://www.un.org/spanish/esa/social/disabled/dis50y20.htm

3. Organización Mundial de la Salud (OMS). "Clasificación Internacional del Funcionamiento, de la Discapacidad y de la Salud "CIF»". Madrid: Ministerio de Trabajo y Asuntos Sociales. Secretaría General de Asuntos Sociales. Instituto de Migraciones y Servicios Sociales (IMSERSO); 2001.

4. Convención sobre los Derechos de las Personas con Discapacidad, Organización de Naciones Unidas, 13 diciembre de 2006. Disponible en: http://www.un.org/spanish/disabilities/convention/convention.html

5. INSTRUMENTO de Ratificación de la Convención sobre los derechos de las personas con discapacidad, hecho en Nueva York el 13 de diciembre de 2006. Boletín Oficial del Estado, no 96 (21-04-2008).

6. Instituto Nacional de Estadística." Encuesta de Discapacidad, Autonomía Personal y situaciones de Dependencia (EDAD)". Notas de Prensa, 4 de noviembre de 2008. Disponible en: http://www.ine.es/prensa/np2008.htm

7. Peláez A, Martínez B, Leonhard M. "Maternidad y discapacidad". 1ª Edic. Madrid: Ediciones Cinca; 2009.

8. Welner S: "Screening Issues in Gynecologic Malignancies for Women with Disabilities: Critical Considerations". J Women's Health 1997; 7: 3-202.

9. Smeltzer SC. "Pregnancy in women with physical disabilities". J Obstet Gynecol Neonatal Nurs. 2007 Jan-Feb; 36(1): 88-96.

10. CERMI (Comité Español de representantes de personas con discapacidad). "Informe ejecutivo", presentado al comité de naciones unidas para la convención sobre la eliminación de todas las formas de discriminación contra la mujer. 2009. Disponible en:

www. boletin.cermi.es/render.aspx?fichero $=113$

11. Nosek, MA, Howland, CA, Rintala, DH, joven, ME, Chanpong, GF "Estudio Nacional de la Mujer con Discapacidad física: informe final". Sexualidad y Discapacidad, 2001; 19 (1): 5-39.

12. Nosek M, Young M, Rintala D, Howland C, Foley C, Bennett, J. "Barriers to reproductive health maintenance among women with physical disabilities". J Women's Health 1995; 4: 505-517.

13. García de la Cruz J J. Zarco J" El espejo social de la mujer con gran discapacidad: barreras sociales para retornar a una vida normal" Editorial Fundamentos. Madrid, 2004.

14. Marriner A, Raile $M$ "Modelos y Teorías en Enfermería". $6^{a}$ ed. Madrid: Elsevier Mosby; 2007.

15. McCall, L. "The Complexity of Intersectionality." Journal of Women in Culture and Society 30.02005 1771-1800. 26 de noviembre de 2007 http://www.journals.uchicago.edu/doi/pdf/10.1086/426800 
16. La Rivière-Zijdel L. Conferencia Europea "Reconociendo los derechos de las niñas y las mujeres con discapacidad". Madrid 2007. CERMI. Edic. Cinca.

17. Heras-García, MA. "Discapacidad y modelo social en el marco de la Convención de la ONU 2006: personas mayores y derecho civil". Madrid Informes Portal Mayores, $\mathrm{n}^{\circ}$ 101. 2010. Disponible en:

http://www.imsersomayores.csic.es/documentos/documentos/heras-discapacidad$\underline{01 . p d f}$

18. Hahn, H. "Hacia una Política de Discapacidad: Definiciones, disciplinas y políticas." 1985. Publicación en Internet URL:

www.independentliving.org/docs4/hahn2.html

19. Hahn H. The Political Implications of Disability Definitions and Data Journal of Disability Policy Studies. 1993; 4: 41-5.

20. López, M."Mujeres con discapacidad. Mitos y realidades en las relaciones de pareja y en la maternidad". Madrid: Edic. Narcea (Colección Mujeres); 2008.

21. Etxeberria X, Flórez J. "Discapacidad y sociedad: aspectos éticos". Disponible en: http://www.down21.org/web n/index.php?option=com content\&view=category\&id=87 8:etica-\&ltemid=2309\&layout=default

22. Stangl R. "Selective terminations and respect for the disabled". J Med Philos. 2010 Feb; 35(1): 32-45. Epub 2009 Dec 24.

23. Martínez H., "Programa de atención integral a la mujer. Consenso" Murcia, Diciembre 2007.

24. Ley Básica reguladora de la autonomía del paciente y de derechos y obligaciones en materia de información y documentación clínica. Ley 41/2002 de 14 de noviembre. Boletín Oficial del Estado, no 274, (15-11-2002).

25. Albrecht $P$, Fischer D, Moser A. "Multiple sclerosis and pregnancy: what does the patient think? a questionnaire study". BMC Res Notes, 2010; 3: 91.

26. Reina M, Adya $M$, Blanck $P$. "Mujeres con discapacidad en el marco legal internacional de derechos humanos". Programa de cooperación sobre derechos humanos. Mexico-Comisión Europea. 2006. Disponible en:

http://portal.sre.gob.mx/pcdh/libreria/libro11/mujeres\%20con\%20discapacidad.pdf.

27. Constitución Española. De 27 de diciembre de 1978. Disponible en: http://www.boe.es/aeboe/consultas/enlaces/documentos/ConstitucionCASTELLANO. $\underline{\mathrm{pdf}}$

28. Ley de Promoción de la Autonomía Personal y Atención a las personas en situación de Dependencia. Ley 39/2006 de 14 de diciembre. Boletín Oficial del Estado, nº 299 (15-12-2006).

29. Ley para la Igualdad efectiva de hombres y mujeres. Ley 3/2007 de 22 de marzo. Boletín Oficial del Estado, no71 (23-03-2007).

30. Ley de investigación bio-médica. Ley 14/2007 de 3 de julio. Boletín Oficial del Estado, no159 (04-07-2007).

31. III Plan de Acción para las Personas con Discapacidad 2009-2012. Gobierno de España y CERMI. Boletín Oficial del Estado no 107, (03-05-2010).

32. Ley de los Derechos y Deberes de los usuarios del sistema sanitario de la Región de Murcia. Ley 3/2009 de 11 de mayo. Boletín Oficial de la Región de Murcia, № 114 (20-05-2009).

33. Jiménez MT, González P, Martín JM. "La clasificación internacional del funcionamiento, de la discapacidad y de la salud (CIF) 2001", Rev. Esp. Salud Publica, 2002; 76(4): 271-279.

34.CIE.9.MC."Clasificación Internacional de Enfermedades 9a revisión Modificación Clínica". $5^{a}$ ed. Madrid: Editado por el Ministerio de Sanidad y Consumo, Secretaria General Técnica. 2006. 
35. Icart MT, Fuentelsaz C, Pulpón A, Elaboración y presentación de un proyecto de investigación y una tesina. Metodología 1. Edicions Universitat de Barcelona. 2001; p. 59.

36. Mayan M. Una introducción a los métodos cualitativos: módulo de entrenamiento para estudiantes y profesionales. Qual Institute Press. International Institute for Qualitative Methodology [en pdf] 2001 [fecha de acceso 14 de junio de 2009]; URL disponible en: http://www.ualberta.ca/ iiqm//pdfs/introduccion.pdf

37. Maderuelo JA. "Cuestionario de satisfacción de las mujeres con el seguimiento del embarazo". Gac Sanit. 2006 Jan-Feb; 20(1): 31-9.

38. De la Cuesta Benjumea C. Desafíos de la investigación cualitativa. Rev Nure Inv [en línea] 2006 [fecha de acceso 4 de septiembre de 2009]; 20. URL disponible en: http://www.nureinvestigacion.es/firma obj.cfm?id firma=37\&ID FIRMA INI=1

39. De la Cuesta Benjumea C. Estrategias cualitativas más usadas en el campo de la salud. Rev Nure Inv [Pdf] 2006 [fecha de acceso 4 de septiembre de 2009]; 25. URL disponible en:

http://www.nureinvestigacion.es/FICHEROS ADMINISTRADOR/F METODOLOGIC A/FMetod 25.pdf

40. Real Decreto de procedimiento para el reconocimiento, declaración y calificación de grado de minusvalía. Real Decreto 1971/1999 de diciembre. Boletín Oficial del Estado, no 22 (26-01-200).

41. Ferrer Arnedo C, Fernández Rodríguez M, Romero Galindo MS, Arana-Echevarria Morales JL, Vielva Asenjo J, Toro Flores $\mathrm{R}$ et al. La intimidad del cuidado y el cuidado de lo íntimo. II Encuentro de Enfermería de la ABFyC: Reflexiones éticas desde Enfermería [pdf] 2005 [fecha de acceso 15 de octubre de 2009]. Disponible en: http://www.asociacionbioetica.com/documentos/Intimidad cuidado.pdf

42. López Espuela et al. "La intimidad de los pacientes percibida por los de Enfermería". Nure Investigación, 2010, n 46, Mayo - Junio, 10.

43. Lumbiganon P, Laopaiboon M, Gülmezoglu AM, Souza JP, Taneepanichskul S, Ruyan P et al. "Method of delivery and pregnancy outcomes in Asia: the WHO global survey on maternal and perinatal health 2007-08". Lancet 2010; 375(9713): 490-9. Citado en PubMed PMID 20071021.

44. Grupo de trabajo de preanestesia del Consorcio Hospital Universitario de Valencia. "Manual de Procedimientos de la Consulta de Preanestesia". Septiembre 2004. Valencia

45. Disability, Pregnancy \& Parenthood international (DPPI). "The maternity information gap for physically disabled peoplea". Junio 2009. Disponible en: http://www.dppi.org.uk/projects/episurvey.php

46. COCEMFE. "Por nosotras y nuestras familias". Manifiesto de las mujeres con discapacidad. (Confederación Coordinadora Estatal de Minusválidos Físicos de España); 2004. Disponible en: http://www.accu.es/accualc/doc/manifies.doc

47. Rogers J."Guía para la mujer con Discapacidad. Del embarazo al parto". Badalona: Fundació Institut Guttman; 2006.

48. Ronald L. Mace, FAIA. "Removing Barriers to Health Care. A Guide for Health Professionals". Ed. Comunicaciones Woodward, North Carolina Comité Asesor en Discapacidad y Salud. Disponible en:

http://www.fpg.unc.edu/ ncodh/rbar/ 
ISSN 1695-6141

(9) COPYRIGHT Servicio de Publicaciones - Universidad de Murcia 